\title{
Predicting Fracture Evolution During Lithiation Process Using Peridynamics
}

\author{
Hanlin Wang, Erkan Oterkus* and Selda Oterkus \\ Department of Naval Architecture, Ocean and Marine Engineering \\ University of Strathclyde \\ 100 Montrose Street Glasgow G4 0LZ, United Kingdom
}

\begin{abstract}
Silicon is regarded as one of the most promising anode materials for lithium-ion batteries due to its large electric capacity. However, silicon experiences large volumetric change during battery cycling which can lead to fracture and failure of lithium-ion batteries. The lithium concentration and anode material phase change have direct influence on hydrostatic stress and damage evolution. High pressure gradient around crack tips causes mass flux of lithium ions which increases the lithium-ion concentration in these regions. Therefore, it is essential to describe the physics of the problem by solving fully coupled mechanical-diffusion equations. In this study, these equations are solved using peridynamics in conjunction with newly introduced peridynamic differential operator concept used to convert partial differential equation into peridynamic form for the diffusion equation. After validating the developed framework, the capability of the current approach is demonstrated by considering a thin electrode plate with multiple pre-existing cracks oriented in different directions. It is shown that peridynamics can successfully predict the crack propagation process during the lithiation process.
\end{abstract}

Keywords: Lithium-ion battery; fracture analysis; peridynamics; phase change; pressure gradient effect

\begin{tabular}{|ll|}
\hline Nomenclature & \\
FEM & Classic Continuum Mechanics \\
PD & Finite Element Method \\
$C$ & Peridynamics \\
$C_{\text {avg }}$ & Current concentration \\
$C_{\mathrm{max}}$ & Average concentration of two material points $\left(\mathrm{mol} / \mathrm{m}^{3}\right)$ \\
$E$ & Maximum lithium ion concentration \\
$G_{\mathrm{c}}$ & Elastic modulus or Young's modulus $\left(\mathrm{N} / \mathrm{m}^{2}\right)$ \\
\hline
\end{tabular}




\begin{tabular}{|c|c|}
\hline$\overline{H_{\mathrm{x}}}$ & Horizon of material point $\mathbf{x}$ \\
\hline$K$ & Fracture toughness $\left(\mathrm{Pa} \cdot \mathrm{m}^{0.5}\right)$ \\
\hline$M$ & Molecular mobility $\left(\mathrm{m}^{2} \mathrm{~J}^{-1} \mathrm{~s}^{-1}\right)$ \\
\hline$N_{\mathrm{A}}$ & Avogadro's constant \\
\hline$T$ & Absolute temperature \\
\hline$\Omega$ & Partial molar volume $\left(\mathrm{m}^{3} \mathrm{~mol}^{-1}\right)$ \\
\hline $\mathbf{b}(\mathbf{x}, t)$ & Body force density of $\mathbf{x}$ at time $t\left(\mathrm{~N} / \mathrm{m}^{3}\right)$ \\
\hline $\mathrm{c}$ & Mechanical bond constant $\left(\mathrm{N} / \mathrm{m}^{6}\right)$ \\
\hline $\mathrm{dV}_{\mathbf{x}^{\prime}}$ & Volume of material particle $\mathbf{x}^{\prime}$ inside the horizon of $\mathbf{x}\left(\mathrm{m}^{3}\right)$ \\
\hline f & Pairwise force density $\left(\mathrm{N} / \mathrm{m}^{6}\right)$ \\
\hline$h$ & Thickness of electrode plate (m) \\
\hline$k_{\mathrm{B}}$ & Boltzmann constant $\left(\mathrm{JK}^{-1}\right)$ \\
\hline$S$ & Bond stretch \\
\hline$s_{\mathrm{c}}$ & Critical bond stretch \\
\hline$\ddot{\mathbf{u}}(\mathbf{x}, t)$ & Acceleration of $\mathbf{x}$ at time $t\left(\mathrm{~m} / \mathrm{s}^{2}\right)$ \\
\hline $\mathbf{u}(\mathbf{x}, t)$ & Displacement of $\mathbf{x}$ at time $t(\mathrm{~m})$ \\
\hline $\mathbf{u}\left(\mathbf{x}^{\prime}, t\right)$ & Displacement of $\mathbf{x}^{\prime}$ at time $t(\mathrm{~m})$ \\
\hline$\alpha$ & Coefficient of expansion \\
\hline$\delta$ & Horizon size (m) \\
\hline$\eta$ & Relative displacement of two material particles (m) \\
\hline$\mu(t, \xi)$ & Failure parameter of bond at time $\mathrm{t}$ \\
\hline$v$ & Poisson's Ratio \\
\hline$\xi$ & Relative position of two material points (m) \\
\hline$\rho$ & Mass density $\left(\mathrm{Kg} / \mathrm{m}^{3}\right)$ \\
\hline$\sigma$ & Cauchy stress $(\mathrm{Pa})$ \\
\hline$\tilde{\sigma}$ & Hydrostatic stress $(\mathrm{Pa})$ \\
\hline
\end{tabular}




\begin{tabular}{|ll|}
\hline$\sigma_{0}$ & First Piola-Kirchoff stress $(\mathrm{Pa})$ \\
$\sigma_{x x}$ & Normal stress along $x$ direction \\
$\sigma_{y y}$ & Normal stress along $y$ direction \\
$\sigma_{x y}, \sigma_{y x}$ & Shear stress in x-y plane \\
$\varphi(\mathrm{x}, \mathrm{t})$ & Damage of material point $\mathbf{x}$ at time $t$ \\
\hline
\end{tabular}

\section{Introduction}

Performance of lithium-ion batteries mainly depends on material properties of anode, cathode and electrolyte. Several metals and compounds are selected as anode material such as cobalt, nickel, manganese and iron phosphate due to their performances in terms of thermal stability, capacity, conductivity and safety [1]. Silicon is also found to be one of the most promising anode materials in lithium-ion batteries due to its high theoretical charging capacity $(3600 \sim 4200 \mathrm{mAHg}-1)$ [2, 3]. However, as lithium ions diffuse into silicon particles, the silicon particles experience a large volume expansion up to around $400 \%[2,4]$. Frequent cycling of the lithium ion battery leads to stress misdistribution, degradation and delamination of the battery components which can significantly affect the battery performance.

Many studies were conducted on fracture analysis of silicon based anode. Liu et al. [5] developed a thin silicon film model to investigate the lithiation induced tensile stress and surface cracking by analytical and finite element methods. They observed a compression-traction transition zone located at lithiated and unlithiated material interface. Large volumetric expansion, plastic deformation and slow charging rate are the main factors which build up this transition zone and lead to cracks and fracture. They also argued that the magnitude and profiles of tensile stress at the surface of lithiated section depends on volumetric misfit strain, yield stress and modulus of unlithiated section.

Stamps and Huang [6] performed a mixed-mode fatigue evaluation of lithium-ion batteries by setting up a pre-cracked electrode model. They found that if a crack can propagate under certain loading when the length of a pre-existing crack is more than half size of the electrode width, the same loading will also cause propagation of a longer pre-existing crack in electrode. On the other hand, Ryu et al. [4] found that pressure gradient always occurs accompanied by a large volume expansion during normal cycling process and this will affect the process of lithium-ion diffusion in the electrode particles and crack evolution. Grantab and Shenoy [7] provided an investigation about pressure gradient factor on crack propagation in silicon nanowires. They used cohesive finite element method to model fracture in nanowires. In the early 
diffusion stage, since localised pressure around the crack tip is lower than surrounding nanowire surface region, large amount of lithium-ions moves into crack tip region which cause relatively large volume expansion and the stress around crack tip eventually reduces. On the other hand, Zuo and Zhao [8] used phase field method to study the stress evolution and crack propagation [8]. A series of damaged electrode models with different crack number and different crack orientations were considered to illustrate the evolution of fracture in an electrode. They indicated that pressure gradient factor depends on Young's modulus, partial molar volume, concentration, Poisson's ratio and the localised concentration around the crack tip region. Gao and Zhou [9] investigated the softening effects caused by lithiation induced fracture in electrode material. They developed a finite element framework and applied J-integral method to investigate the propagation of the crack tip. They also observed large amount of lithium-ion accumulation at the crack tip regions during charging process which causes a relaxation of hydrostatic stress.

As an alternative approach to finite element method (FEM), peridynamics (PD) can be utilised. Peridynamic theory is a new continuum mechanics formulation introduced by Silling [19] to overcome the problems that Classical Continuum Mechanics encountered especially for predicting crack initiation and propagation. Peridynamic theory is based on integro-diffential equations and these equations do not contain any spatial derivatives. Since its introduction, there has been a rapid progress on peridynamics. Several novel approaches have been proposed for efficient numerical solution of peridynamic equations such as dual-horizon concept [20] and adaptive refinement [21]. The technique has been applied to many different material systems $[22,23]$ and extended for the analysis of multifield problems [24,25]. An extensive review on peridynamics can be found in Madenci and Oterkus [26].

In this study, peridynamic theory is used to investigate the fracture evolution in electrode plates of lithium ion batteries by considering pressure gradient and material phase change factors. Coupled field equations are expressed by using a combination of bond-based peridynamics [19] and peridynamic differential operator concept [10] to represent the relationships between the lithium ion concentration, hydrostatic stress and mechanical deformation. Several numerical cases with different crack numbers and crack orientations are considered and analysed.

\section{Coupled diffusion-mechanical deformation mechanism}

The general lithium diffusion phenomenon can be represented by using Fick's Second Law [28]. Since silicon is selected as anode material for lithium ion battery in this study, there is a large amount of volume change during lithiation and delithiation processes. Therefore, the stress induced by volume change during battery cycling should not be ignored. The stress components, $\sigma_{i j}$, can be expressed as [8]

$$
\sigma_{x x}=\frac{E}{1-v^{2}}\left(\frac{\partial u_{x}}{\partial x}+v \frac{\partial u_{y}}{\partial y}\right)-\frac{E \Omega C_{\max }}{3(1-v)} C
$$




$$
\begin{aligned}
& \sigma_{y y}=\frac{E}{1-v^{2}}\left(\frac{\partial u_{y}}{\partial y}+v \frac{\partial u_{x}}{\partial x}\right)-\frac{E \Omega C_{\max }}{3(1-v)} C \\
& \sigma_{x y}=\sigma_{y x}=\frac{E}{2(1+v)}\left(\frac{\partial u_{x}}{\partial y}+\frac{\partial u_{y}}{\partial x}\right)
\end{aligned}
$$

where $\Omega$ is the partial molar volume of silicon, $u_{i}$ is the displacement, $C_{\max }$ is the maximum lithium concentration in the plate and $C$ is the current normalised concentration. As lithiation proceeds in the electrode, local stresses will increase at high geometrical singularity region such as crack tips. Due to the pressure-gradient, large amount of lithium ions move into these regions which will lead to relatively large volume expansion and increase of lithium ion concentration. By considering these factors, the general Fick's Second Law should be modified as [8]

$$
\frac{\partial C}{\partial t}=M k_{B} T\left(\frac{\partial^{2} C}{\partial x^{2}}+\frac{\partial^{2} C}{\partial y^{2}}\right)-\frac{M C \Omega}{N_{A}}\left(\frac{\partial^{2} \tilde{\sigma}}{\partial x^{2}}+\frac{\partial^{2} \tilde{\sigma}}{\partial y^{2}}\right)-\frac{M C \Omega}{N_{A}}\left(\frac{\partial C}{\partial x} \frac{\partial \tilde{\sigma}}{\partial x}+\frac{\partial C}{\partial y} \frac{\partial \tilde{\sigma}}{\partial y}\right)
$$

where $M$ is molecular mobility, $k_{B}$ is Boltzmann constant, $T$ is absolute temperature, $N_{A}$ is Avogadro's constant and $\widetilde{\sigma}$ is the hydrostatic stress.

Once the lithium-ions diffuse into the anode plate, the anode material transforms from pure silicon to lithiated silicon $\left(\mathrm{Li}_{\mathrm{x}} \mathrm{Si}\right)$. Normally, the material properties of anode plate, such as elastic modulus and fracture toughness, will decrease depending on the state of lithiation. Hence, the silicon lithiation process is also known as material softening process [11]. Zhang et al. [12] have a detailed investigation about silicon lithiation on battery electrode. They found that silicon experiences partial and fully lithiation processes. The partially liathiated silicon $\left(\mathrm{Li}_{\mathrm{x}} \mathrm{Si}\right)$ layer lies between fully lithiated silicon $\left(\mathrm{Li}_{15} \mathrm{Si}_{4}\right)$ and pure Silicon regions. However, in this study, since the thickness of this transition layer is very thin compared to the anode geometry, this interface layer is not taken into consideration.

\section{Peridynamic theory}

For the numerical solution of the problem, peridynamic theory [13] was utilised. Peridynamics is a new continuum mechanics formulation and it is very suitable to predict fracture initiation and propagation due to its mathematical structure. It is also advantageous to solve moving boundary problems as in the lithiation process considered in this study which is difficult to handle by using traditional techniques due to necessity of remeshing, etc. Due to its non-local characteristic [14], each material point can build up interactions with other material points within a certain distance $\delta$ (as shown in Fig.1). For material points outside this domain, it is assumed the interactions are too weak so that they can be ignored. Hence, all material points inside the domain build up the horizon $\left(H_{x}\right)$ of the material point $\mathbf{x}$. Each interaction can be considered as a bond and the equation of motion of each material point can be written according to Newton's Second Law as 


$$
\rho \ddot{\mathbf{u}}(\mathbf{x}, t)=\int_{H_{\mathbf{x}}} \mathbf{f}\left(\mathbf{u}\left(\mathbf{x}^{\prime}, t\right)-\mathbf{u}(\mathbf{x}, t), \mathbf{x}^{\prime}-\mathbf{x}\right) d V_{\mathbf{x}^{\prime}}+\mathbf{b}(\mathbf{x}, t)
$$

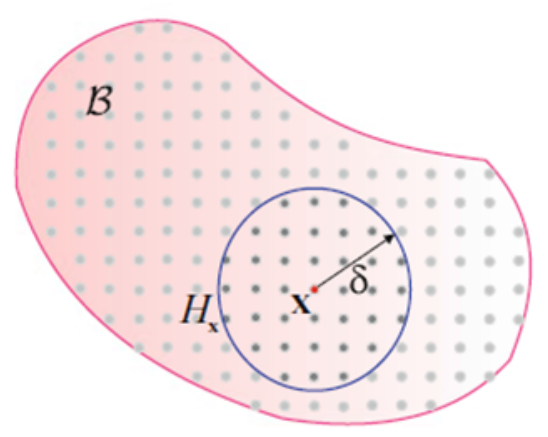

Figure 1. Horizon of material point $\mathbf{x}$

where $\mathbf{b}$ refers to the body force density and $\mathbf{f}$ is the pairwise force of each bond. According to bond-based peridynamic formulation used in this study, the bond force depends on the relative position and relative displacement of material points associated with this bond.

The relative position of material points, $\mathbf{x}$ and $\mathbf{x}^{\prime}$, can be expressed from the original coordinates of these points as

$\xi=\mathbf{x}^{\prime}-\mathbf{x}$

and the relative displacement can be calculated as

$\eta=\mathbf{u}\left(\mathbf{x}^{\prime}, t\right)-\mathbf{u}(\mathbf{x}, t)$

Hence, the stretch of a bond can be defined as

$$
s=\frac{|\xi+\eta|-|\xi|}{|\xi|}
$$

The pairwise force for bond-based peridynamics can be expressed as [17]

$\mathbf{f}=\frac{\xi+\eta}{|\xi+\eta|} c\left(s-\alpha C_{a v g}\right) \mu(t, \xi)$

where $\alpha$ is the coefficient of expansion and $C_{a v g}$ represents the average lithium-ion concentration of material points $\mathbf{x}$ and $\mathbf{x}^{\prime}, \mu$ is the failure parameter and $c$ is the bond constant. For two-dimensional problems and based on plane stress assumption, the bond constant can be related to Elastic modulus, $E$ as

$c=\frac{9 E}{\pi h \delta^{3}}$ 
where $h$ is the thickness of the plate. Note that for bond-based peridynamics, Poisson's ratio is constant and equal to $v=1 / 3$ for plane stress condition.

For an elastic brittle material, it can be assumed that once a bond exceeds a critical stretch value, $s_{c}$ after deformation, it will break and cannot recover. The critical stretch in peridynamic theory can be related to critical strain energy release rate. For two dimensional problems and based on plane stress assumption, critical stretch can be expressed in terms of critical strain energy release rate as

$$
s_{c}=\frac{2}{3} \sqrt{\frac{\pi G_{c}}{E \delta}}
$$

The failure condition of each bond can be represented by a failure parameter as

$$
\mu(t, \xi)= \begin{cases}1 & s \leq s_{c} \\ 0 & s>s_{c}\end{cases}
$$

Once a bond breaks, the mechanical load acting on material point $\mathbf{x}$ will be redistributed on remaining bonds within its horizon and mechanical condition of this point will change. The local damage of the material point $\mathbf{x}$ can be defined as

$$
\varphi(\mathbf{x}, t)=1-\frac{\int_{H_{\mathbf{x}}} \mu(t, \xi) d V_{\mathbf{x}^{\prime}}}{\int_{H_{\mathbf{x}}} d V_{\mathbf{x}^{\prime}}}
$$

Local damage is the percentage of broken bonds associated with material point $\mathbf{x}$. Local damage varies from 0 to 1 where 0 represents undamaged and 1 refers to fully damaged material point.

As mentioned earlier, the lithium-ion concentration depends on the hydrostatic stress, $\tilde{\sigma}$. For two dimensional problems and based on plane stress assumption, hydrostatic stress of a material point $\mathbf{x}$ can be calculated as [15]

$$
\tilde{\sigma}=\frac{1}{2}\left(\sigma_{x x}+\sigma_{y y}\right)
$$

Cauchy stress tensor, $\sigma_{i j}$, can be written in terms first Piola-Kirchoff stress, $\sigma_{0}$, as

$$
\sigma=J \sigma_{0} F^{T}=\left[\begin{array}{ll}
\sigma_{x x} & \sigma_{x y} \\
\sigma_{y x} & \sigma_{y y}
\end{array}\right]
$$

where $F$ is the deformation gradient and $J$ is defined as

$$
J=\operatorname{det}(F)
$$


The stress definitions are not directly used in peridynamics. However, they can be related to peridynamic parameters. Therefore, the first Piola-Kirchoff stress, $\sigma_{0}$, can be calculated as [18]

$$
\sigma_{0}=\int_{H_{\mathbf{x}}} \mathbf{f} \otimes\left(\mathbf{x}^{\prime}-\mathbf{x}\right) d V_{\mathbf{x}^{\prime}}
$$

Moreover, the deformation gradient, $F$, can also be defined in peridynamic framework as [18]

$$
F=\frac{2}{\pi h \delta^{2}} \int_{H_{\mathbf{x}}} \frac{1}{|\xi|^{2}}(\xi+\eta) \otimes \xi d V_{\mathbf{x}^{\prime}}
$$

By recalling Eqs. (1) and (2), the mechanical stresses depend on the displacement gradients and lithium-ion concentration, while the concentration change rate depends on the hydrostatic stress gradient and lithium-ion concentration gradient. In this study, the peridynamic form of partial differential equation given in Eq. (2) is obtained by using peridynamic differential operator method developed by Madenci et al. [10].

\section{Peridynamic differential operator approach}

Similar to traditional finite differential schemes, peridynamic differential operator can be derived from Taylor Series [10]

$$
f\left(\mathbf{x}^{\prime}\right)=f(\mathbf{x})+\xi_{1} \frac{\partial f(\mathbf{x})}{\partial x_{1}}+\xi_{2} \frac{\partial f(\mathbf{x})}{\partial x_{2}}+\frac{1}{2 !} \xi_{1}^{2} \frac{\partial^{2} f(\mathbf{x})}{\partial x_{1}^{2}}+\frac{1}{2 !} \xi_{2}^{2} \frac{\partial^{2} f(\mathbf{x})}{\partial x_{2}^{2}}+\xi_{1} \xi_{2} \frac{\partial^{2} f(\mathbf{x})}{\partial x_{1} \partial x_{2}}+R
$$

Eq. (17) is a two dimensional second order form of Taylor Series and $R$ represents the remainder terms which is small enough to be neglected. $f$ is a function which depends on the problem considered. Moving the first term on the right hand side of Eq. (17) to the left hand side, multiplying each term with a peridynamic function $g_{2}^{p_{1} p_{2}}(\xi)$ (with $\mathrm{p}_{1}, \mathrm{p}_{2}=0,1,2$ except $\left.\mathrm{p}_{1}=\mathrm{p}_{2}=0\right)$ and integrating throughout the horizon, Eq. (17) will take a new form of

$$
\begin{aligned}
& \int_{H_{\mathbf{x}}}\left[f\left(\mathbf{x}^{\prime}\right)-f(\mathbf{x})\right] g_{2}^{p_{1} p_{2}}(\xi) d V_{\mathbf{x}^{\prime}}= \\
& \frac{\partial f(\mathbf{x})}{\partial x_{1}} \int_{H_{\mathbf{x}}} \xi_{1} g_{2}^{p_{1} p_{2}}(\xi) d V_{\mathbf{x}^{\prime}}+\frac{\partial f(\mathbf{x})}{\partial x_{2}} \int_{H_{\mathbf{x}}} \xi_{2} g_{2}^{p_{1} p_{2}}(\xi) d V_{\mathbf{x}^{\prime}}+\frac{\partial^{2} f(\mathbf{x})}{\partial x_{1}^{2}} \int_{H_{\mathbf{x}}} \frac{1}{2 !} \xi_{1}^{2} g_{2}^{p_{1} p_{2}}(\xi) d V_{\mathbf{x}^{\prime}} \\
& +\frac{\partial^{2} f(\mathbf{x})}{\partial x_{2}^{2}} \int_{H_{\mathbf{x}}} \frac{1}{2 !} \xi_{2}^{2} g_{2}^{p_{1} p_{2}}(\xi) d V_{\mathbf{x}^{\prime}}+\frac{\partial^{2} f(\mathbf{x})}{\partial x_{1} \partial x_{2}} \int_{H_{\mathbf{x}}} \xi_{1} \xi_{2} g_{2}^{p_{1} p_{2}}(\xi) d V_{\mathbf{x}^{\prime}}
\end{aligned}
$$

The orthogonality property of the peridynamic function can be written as [10] 
$\frac{1}{n_{1} ! n_{2} !} \int_{H_{\mathbf{x}}} g_{2}^{p_{1} p_{2}}(\xi) d V_{\mathbf{x}^{\prime}}=\bar{\delta}_{n_{1} p_{1}} \bar{\delta}_{n_{2} p_{2}}$ with $\mathrm{n}_{1}=\mathrm{n}_{2}=0,1,2$

where $\bar{\delta}$ is the Kronecker delta. By utilising Eq. (19), partial derivatives can be expressed in peridynamic form as

$$
\left.\left.\left\{\begin{array}{c}
\frac{\partial f(\mathbf{x})}{\partial x_{1}} \\
\frac{\partial f(\mathbf{x})}{\partial x_{2}} \\
\frac{\partial^{2} f(\mathbf{x})}{\partial x_{1}^{2}} \\
\frac{\partial^{2} f(\mathbf{x})}{\partial x_{2}^{2}} \\
\frac{\partial^{2} f(\mathbf{x})}{\partial x_{1} \partial x_{2}}
\end{array}\right\}=\int_{H_{\mathbf{x}}}\left[f\left(\mathbf{x}^{\prime}\right)-f(\mathbf{x})\right]\right\} \begin{array}{l}
g_{2}^{10}(\xi) \\
g_{2}^{01}(\xi) \\
g_{2}^{20}(\xi) \\
g_{2}^{02}(\xi) \\
g_{2}^{11}(\xi)
\end{array}\right\} d V_{\mathbf{x}^{\prime}}
$$

The peridynamic functions $g_{2}^{p_{1} p_{2}}(\xi)$ in Eq. (20) can be constructed by polynomials which contain a weight function $\omega$ and unknown coefficients $a$ as [10]

$$
\begin{aligned}
& g_{2}^{p_{1} p_{2}}(\xi)=a_{10}^{p_{1} p_{2}} \omega_{10}(|\xi|) \xi_{1}+a_{01}^{p_{1} p_{2}} \omega_{01}(|\xi|) \xi_{2}+a_{20}^{p_{1} p_{2}} \omega_{20}(|\xi|) \xi_{1}^{2} \\
& +a_{02}^{p_{1} p_{2}} \omega_{02}(|\xi|) \xi_{2}^{2}+a_{11}^{p_{1} p_{2}} \omega_{11}(|\xi|) \xi_{1} \xi_{2}
\end{aligned}
$$

The unknown coefficient matrix components $a$ depends on a peridynamic shape matrix $\mathbf{A}$ and coefficient matrix $\mathbf{b}$ with a relationship given as

$$
\sum_{q_{1}=0}^{2} \sum_{q_{2}=0}^{2-q_{1}} A_{\left(n_{1} n_{2}\right)\left(q_{1} q_{2}\right)} a_{q_{1} q_{2}}^{p_{1} p_{2}}=b_{n_{1} n_{2}}^{p_{1} p_{2}}
$$

in which $q_{1}$ and $q_{2}$ should not be zero at the same time.

The peridynamic shape matrix A depends on the weight functions $\omega$ and components of the relative position of interacting material points associated with a bond, $\xi_{1}, \xi_{2}$. The relationship between these parameters is given as

$$
A_{\left(n_{1} n_{2}\right)\left(q_{1} q_{2}\right)}=\int_{H_{x}} \omega_{q_{1} q_{2}}(|\xi|) \xi_{1}^{n_{1}+p_{1}} \xi_{2}^{n_{2}+p_{2}} d V_{\mathbf{x}^{\prime}}
$$

The coefficient matrix $\mathbf{b}$ can be constructed as

$$
b_{n_{1} n_{2}}^{p_{1} p_{2}}=n_{1} ! n_{2} ! \bar{\delta}_{n_{1} p_{1}} \bar{\delta}_{n_{2} p_{2}}
$$

and the weight function is given as 
$\omega_{q_{1} q_{2}}=\left(\frac{\delta}{|\xi|}\right)^{q_{1}+q_{2}+1}$

For two dimensional problems, the peridynamic shape matrix $\mathbf{A}$ can be written based on Eq. (23) as

$\left[\begin{array}{ccccc}A_{(10)(10)} & A_{(10)(01)} & A_{(10)(20)} & A_{(10)(02)} & A_{(10)(11)} \\ A_{(01)(10)} & A_{(01)(01)} & A_{(01)(20)} & A_{(01)(02)} & A_{(01)(11)} \\ A_{(20)(10)} & A_{(20)(01)} & A_{(20)(20)} & A_{(20)(02)} & A_{(20)(11)} \\ A_{(02)(10)} & A_{(02)(01)} & A_{(02)(20)} & A_{(02)(02)} & A_{(02)(11)} \\ A_{(11)(10)} & A_{(11)(01)} & A_{(11)(20)} & A_{(11)(02)} & A_{(11)(11)}\end{array}\right]=\left[\begin{array}{cccccc}\frac{1}{2} \pi h \delta^{4} & 0 & 0 & 0 & 0 \\ 0 & \frac{1}{2} \pi h \delta^{4} & 0 & 0 & 0 \\ 0 & 0 & \frac{1}{4} \pi h \delta^{6} & \frac{1}{12} \pi h \delta^{6} & 0 \\ 0 & 0 & \frac{1}{12} \pi h \delta^{6} & \frac{1}{4} \pi h \delta^{6} & 0 \\ 0 & 0 & 0 & 0 & \frac{1}{12} \pi h \delta^{6}\end{array}\right]$

The unknown coefficient matrix a can be expressed as

$$
[a]=\left[\begin{array}{ccccc}
a_{10}^{10} & a_{10}^{01} & a_{10}^{20} & a_{10}^{02} & a_{10}^{11} \\
a_{01}^{10} & a_{01}^{01} & a_{01}^{20} & a_{01}^{02} & a_{01}^{11} \\
a_{20}^{10} & a_{20}^{01} & a_{20}^{20} & a_{20}^{02} & a_{20}^{11} \\
a_{02}^{10} & a_{02}^{01} & a_{02}^{20} & a_{02}^{02} & a_{02}^{11} \\
a_{11}^{10} & a_{11}^{01} & a_{11}^{20} & a_{11}^{02} & a_{11}^{11}
\end{array}\right]
$$

According to Eq. (24), the known coefficient matrix b can be calculated as

$$
[b]=\left[\begin{array}{ccccc}
b_{10}^{10} & 0 & 0 & 0 & 0 \\
0 & b_{01}^{01} & 0 & 0 & 0 \\
0 & 0 & b_{20}^{20} & 0 & 0 \\
0 & 0 & 0 & b_{02}^{02} & 0 \\
0 & 0 & 0 & 0 & b_{11}^{11}
\end{array}\right]=\left[\begin{array}{ccccc}
1 & 0 & 0 & 0 & 0 \\
0 & 1 & 0 & 0 & 0 \\
0 & 0 & 2 & 0 & 0 \\
0 & 0 & 0 & 2 & 0 \\
0 & 0 & 0 & 0 & 1
\end{array}\right]
$$

After expanding the summation term in Eq. (22), the relationship between the elements of the shape coefficient matrix A, unknown coefficient matrix a and known coefficient matrix $\mathbf{b}$ can be expressed as:

$$
\begin{aligned}
& A_{(10)(10)} a_{10}^{10}=b_{10}^{10} \\
& A_{(01)(01)} a_{01}^{01}=b_{01}^{01} \\
& A_{(20)(02)} a_{20}^{20}+A_{(20)(02)} a_{02}^{20}=b_{20}^{20}
\end{aligned}
$$


$A_{(02)(20)} a_{20}^{02}+A_{(02)(02)} a_{02}^{02}=b_{02}^{02}$

$A_{(11)(11)} a_{11}^{11}=b_{11}^{11}$

Solving the system of equations given in Eqs. (29a-e), the unknown coefficient matrix a can be obtained as

$[a]=\left[\begin{array}{ccccc}\frac{2}{\pi h \delta^{4}} & 0 & 0 & 0 & 0 \\ 0 & \frac{2}{\pi h \delta^{4}} & 0 & 0 & 0 \\ 0 & 0 & \frac{9}{\pi h \delta^{6}} & -\frac{3}{\pi h \delta^{6}} & 0 \\ 0 & 0 & -\frac{3}{\pi h \delta^{6}} & \frac{9}{\pi h \delta^{6}} & 0 \\ 0 & 0 & 0 & 0 & \frac{12}{\pi h \delta^{6}}\end{array}\right]$

Substituting Eq. (30) into Eq. (21), the peridynamic functions can be calculated as

$g_{2}^{10}(\xi)=\frac{2}{\pi h|\xi| \delta^{2}} \cos \theta$

$g_{2}^{01}(\xi)=\frac{2}{\pi h|\xi| \delta^{2}} \sin \theta$

$g_{2}^{20}(\xi)=\frac{9}{\pi h|\xi| \delta^{3}} \cos ^{2} \theta-\frac{3}{\pi h|\xi| \delta^{3}} \sin ^{2} \theta$

$g_{2}^{02}(\xi)=-\frac{3}{\pi h|\xi| \delta^{3}} \cos ^{2} \theta+\frac{9}{\pi h|\xi| \delta^{3}} \sin ^{2} \theta$

$g_{2}^{11}(\xi)=\frac{12}{\pi h|\xi| \delta^{3}} \cos \theta \sin \theta$

where $\theta$ is the angle between the bond and the horizontal axis. By substituting Eqs. (31a-e) into Eqs. (20), the two dimensional partial derivatives in both first and second order can be calculated. Therefore, the general Fick's Second Law given in Eq. (2) can be written in peridynamic form as 


$$
\begin{aligned}
\frac{\partial C}{\partial t} & =M k_{B} T \int_{H_{\mathbf{x}}}\left\{C\left(\mathbf{x}^{\prime}, t\right)-C(\mathbf{x}, t)\right\}\left\{g_{2}^{20}(\xi)+g_{2}^{02}(\xi)\right\} d V_{\mathbf{x}^{\prime}} \\
& -\frac{M C \Omega}{N_{A}} \int_{H_{\mathbf{x}}}\left\{\tilde{\sigma}\left(\mathbf{x}^{\prime}, t\right)-\tilde{\sigma}(\mathbf{x}, t)\right\}\left\{g_{2}^{20}(\xi)+g_{2}^{02}(\xi)\right\} d V_{\mathbf{x}^{\prime}} \\
& -\frac{M C \Omega}{N_{A}}\left\{\left(\int_{H_{\mathbf{x}}}\left\{C\left(\mathbf{x}^{\prime}, t\right)-C(\mathbf{x}, t)\right\} g_{2}^{10}(\xi) d V_{\mathbf{x}^{\prime}}\right)\left(\int_{H_{\mathbf{x}}}\left\{\tilde{\sigma}\left(\mathbf{x}^{\prime}, t\right)-\tilde{\sigma}(\mathbf{x}, t)\right\} g_{2}^{10}(\xi) d V_{\mathbf{x}^{\prime}}\right)\right\} \\
- & \frac{M C \Omega}{N_{A}}\left\{\left(\int_{H_{\mathbf{x}}}\left\{C\left(\mathbf{x}^{\prime}, t\right)-C(\mathbf{x}, t)\right\} g_{2}^{01}(\xi) d V_{\mathbf{x}^{\prime}}\right)\left(\int_{H_{\mathbf{x}}}\left\{\tilde{\sigma}\left(\mathbf{x}^{\prime}, t\right)-\tilde{\sigma}(\mathbf{x}, t)\right\} g_{2}^{01}(\xi) d V_{\mathbf{x}^{\prime}}\right)\right\}
\end{aligned}
$$

Finally, flow chart for the entire solution process of coupled peridynamic equation of motion given in Eq. (3) and peridynamic differential operator based diffusion equation given in Eq. (32) is depicted in Fig. (2). 


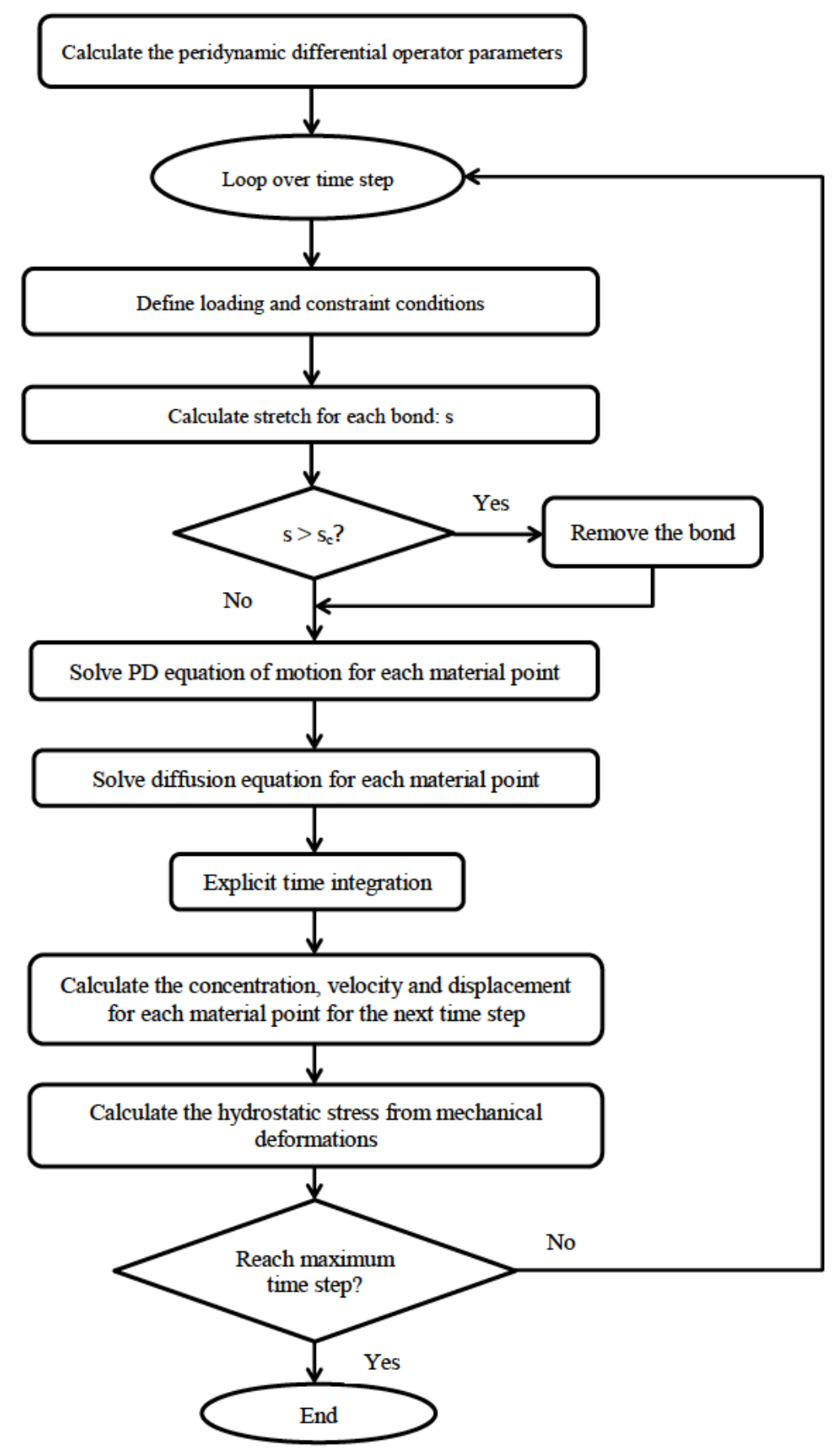

Figure 2. Flow chart for the solution process 


\section{Validation study}

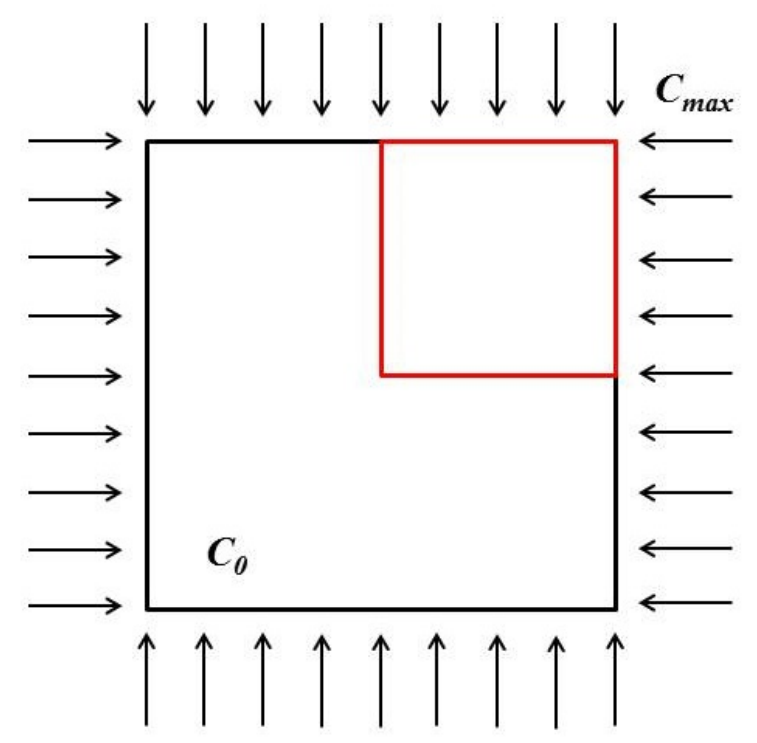

Figure 3. Two-dimensional square plate specimen

In order to validate the peridynamic differential operator approach used in this study, a two-dimensional thin square plate is considered as shown in Fig. (3). Since damage is not considered in this example, the effect of mechanical field on concentration field will be insignificant. Therefore, only the effect of concentration field on mechanical field is taken into account. The plate is initially free from lithium ions. Therefore, initial lithium ion concentration of the plate is $\mathrm{C}_{0}\left(0 \mathrm{~mol} / \mathrm{m}^{3}\right)$. Then, the plate is subjected to a maximum lithium ion concentration, $\mathrm{C}_{\max }\left(0.15 \mathrm{~mol} / \mathrm{m}^{3}\right)$, along its external boundaries. The plate is free from any displacement constraints. An imaginary material, $A$, is considered for simplicity and material properties are given in Table 1 . Since the plate geometry and loading condition are symmetric, only a quarter of the plate is modelled as shown in red contour in Fig. (3). Plane stress assumption is utilized and boundary conditions are imposed by introducing a fictitious region with a thickness equivalent to the horizon size. Dynamic analysis is performed using a time step size of $7.3 \times 10^{-8} \mathrm{~s}$ and the analysis is run for 20000 time steps. Peridynamic results are compared against finite element solutions obtained using a commercially available software, ANSYS. As shown in Fig. (4), both concentration and displacement results from PD and FEM analyses yield similar results which verifies the combined peridynamics and peridynamic differential operator approach used throughout this study. 
Table 1. Material properties for validation case

\begin{tabular}{c|ccccc} 
Material & $\begin{array}{c}\text { Elastic } \\
\text { modulus(GPa) }\end{array}$ & $\begin{array}{c}\text { Poisson's } \\
\text { ratio }\end{array}$ & $\begin{array}{c}\text { Coefficient } \\
\text { of expansion } \\
\left(\mathrm{m}^{3} / \mathrm{mol}\right)\end{array}$ & Density $\left(\mathrm{kg} / \mathrm{m}^{3}\right)$ & $\begin{array}{c}\text { Diffusivity } \\
\left(\mathrm{m}^{2} / \mathrm{s}\right)\end{array}$ \\
\hline$A$ & 200 & $\frac{1}{3}$ & $50 \times 10^{-6}$ & 1 & 1000
\end{tabular}
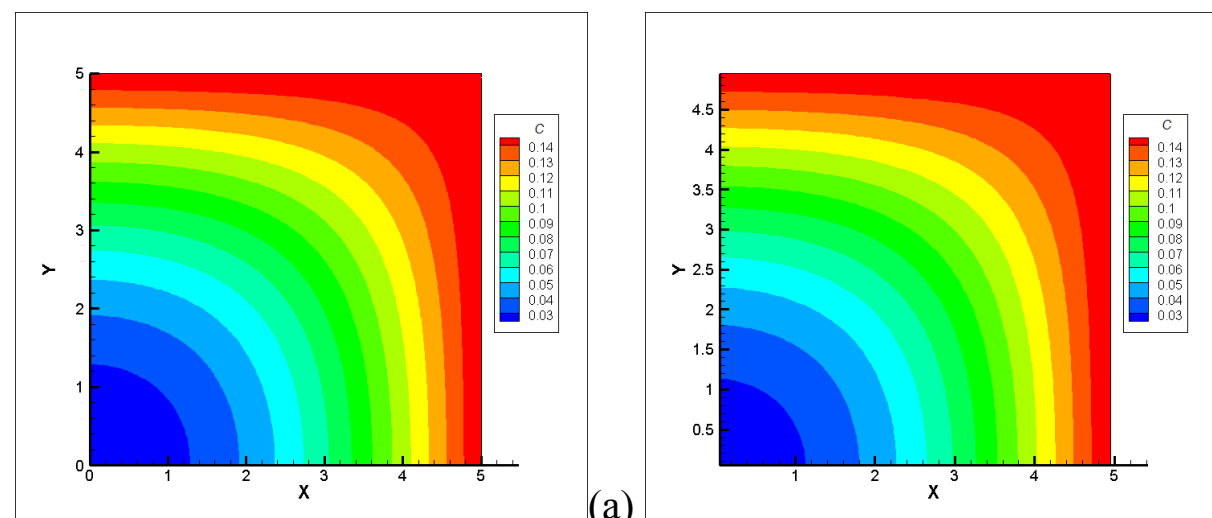

(b)
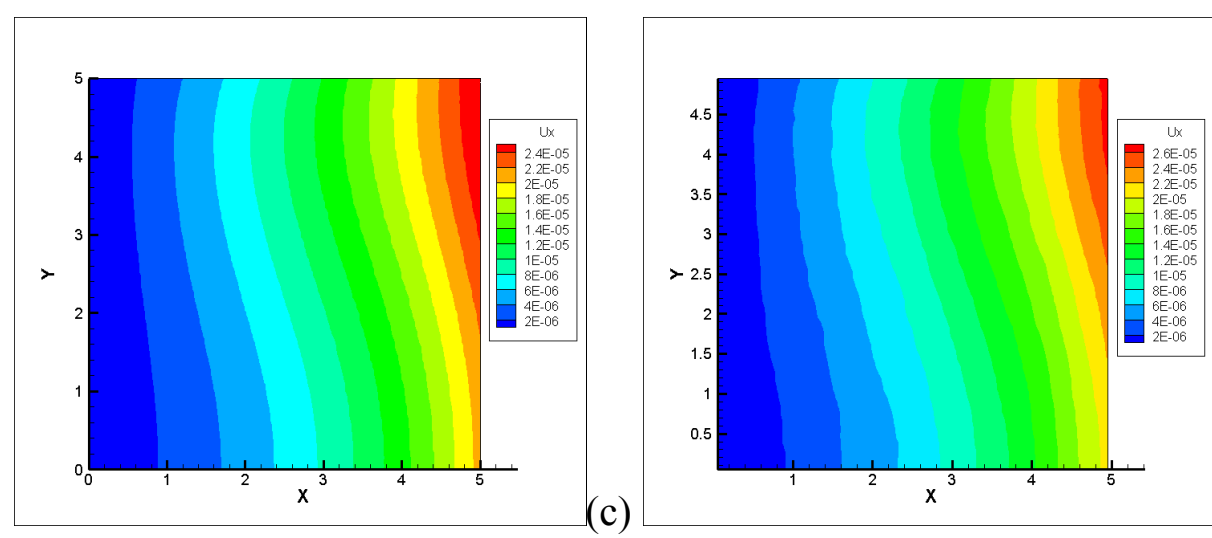

(c)

(d)
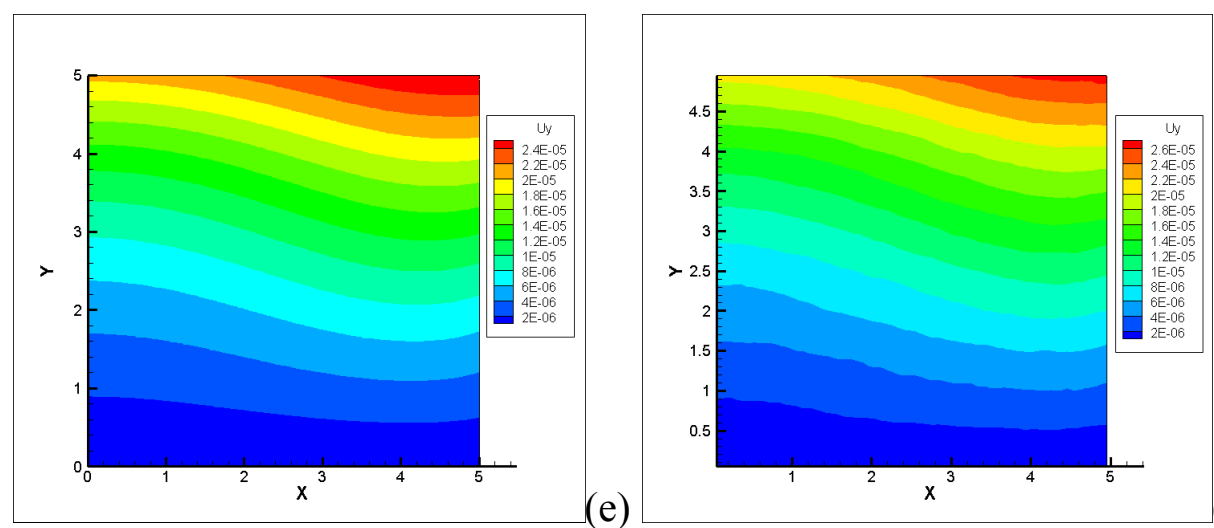

Figure 4. Comparison of PD results against FEM results: (a) Lithium ion concentration distribution by FEM (b) Lithium ion concentration distribution by PD

(c) Displacement in $x$ direction by FEM (d) Displacement in $x$ direction by PD (e) Displacement in $y$ direction by FEM (f) Displacement in $y$ direction by PD. 


\section{Numerical studies}

To demonstrate the capability of the current approach, a square silicon plate is selected to represent the anode plate of the lithium ion battery based on the study considered by Zuo and Zhao [8]. Both pure silicon and lithiated silicon are regarded as brittle materials. Concentration values are normalised by maximum concentration. The thickness of the specimen plate is negligible compared with boundary length. Therefore, plane stress assumption is utilised in order to simplify the numerical simulation. The square specimen is free from lithium-ions at its initial state (see Fig. 5). The silicon specimen is subjected to maximum lithium-ion concentration on all of its boundaries by introducing a fictitious region with a thickness of horizon size along its boundaries [29]. The plate is free from any displacement constraints. Geometrical and material information are summarised in Table 2. Note that bond-based peridynamics is used in this study which enforces a constraint condition on Poisson's ratio, i.e. $v=1 / 3$, which is higher than the actual Poisson's ratio values given for silicon and lithiated silicon. For the cases which are considered in this study, the effect of Poisson's ratio on the fracture pattern is not significant. However, if required, actual Poisson's ratio values can be specified using ordinary state based peridynamic formulation for the equation of motion [27].

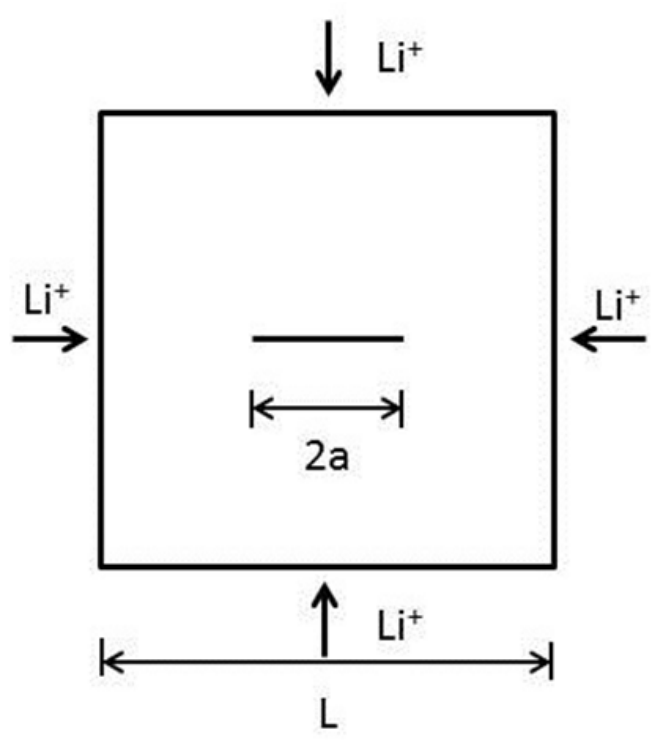

Figure 5. Pre-cracked square electrode plate specimen 
Table 2. Geometrical and material parameters

\begin{tabular}{ccc}
\hline$L$ & Length of the specimen & $1 \mu \mathrm{m}$ \\
$E_{\text {silicon }}$ & Elastic constant of silicon & $80 \mathrm{GPa}$ \\
$E_{L_{15} S i_{4}}$ & Elastic constant of amorphous $\mathrm{Li}_{15} \mathrm{Si}_{4}$ & $41 \mathrm{GPa}$ \\
$v_{\text {silicon }}$ & Poisson's ratio of silicon & 0.22 \\
$v_{L i_{15} S i_{4}}$ & Poisson's ratio of silicon & 0.24 \\
$\Omega$ & Partial molar volume & $8.5 \times 10^{-6} \mathrm{~m}^{3} \mathrm{~mol}^{-1}$ \\
$M$ & Molecular mobility & $500 \mathrm{~m}^{2} \mathrm{~J}^{-1} \mathrm{~s}^{-1}$ \\
$k_{B}$ & Boltzmann constant & $1.38 \times 10^{-23} \mathrm{~J}^{-\mathrm{K}^{-1}}$ \\
$T$ & Absolute temperature & $300 \mathrm{~K}$ \\
$N_{A}$ & Avogadro's constant & $6.02 \times 10^{23} \mathrm{~mol}^{-1}$ \\
$S_{c_{-} S i}$ & Critical strain for silicon & 0.04 \\
$S_{C_{-} L i_{15} S i_{4}}$ & Critical strain for amorphous $\mathrm{Li}_{15} \mathrm{Si}_{4}$ & 0.035 \\
$C_{\max }$ & & $1.18 \times 10^{4} \mathrm{molm}^{-3}$ \\
\hline
\end{tabular}

\subsection{Coupled analysis for a single crack case}

In this case study, an initial crack which has a length equivalent to $20 \%$ of the length of the specimen plate lies horizontally in the central region. Initial damage is shown in Fig. (6a). As lithiation progresses, material at the boundary region of the specimen will expand first. Due to mechanical deformation, compressive stresses form at the boundary region which leads to tension stresses at the central part of the plate. Since crack is located at the central region, stresses concentrate at two crack tip regions which eventually increases the hydrostatic stress (Fig. 6d). According to Eq. (2), high pressure gradient at crack tip regions is one of the main motivations of lithium-ion enrichment. Therefore, as shown in Fig. (6c), the concentration of lithium-ions at crack tip regions is higher than the surrounding regions. Hence, the material of the particles in these regions will transform from pure silicon to lithiated silicon and experience a large volume expansion as described in Eq. (1). As mentioned earlier, once the bond of a particle exceeds the critical value, it will be broken and the interaction will never recover. Large amount of bonds around crack tips are broken after the deformation which leads to crack propagation as shown in Fig. (6b). 

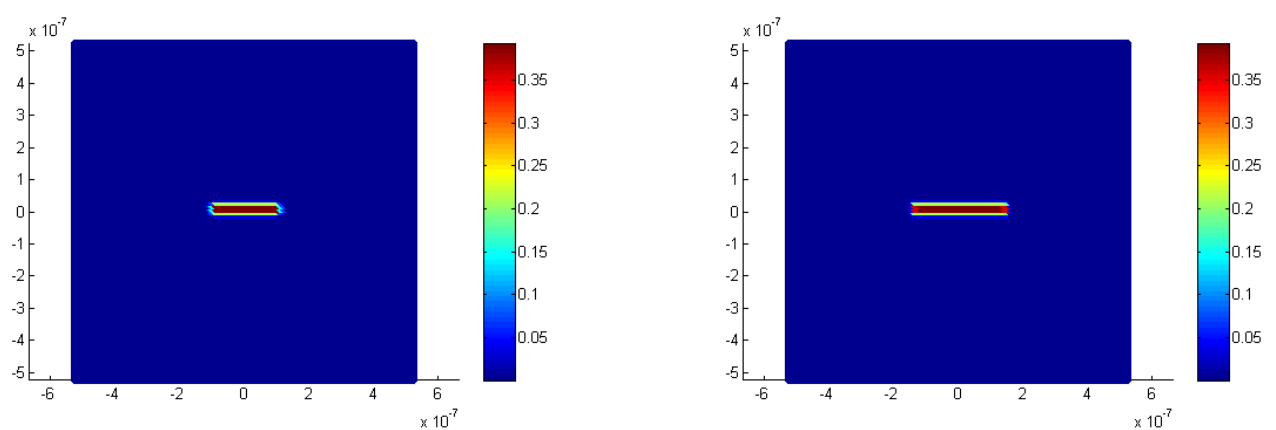

(a)

(b)
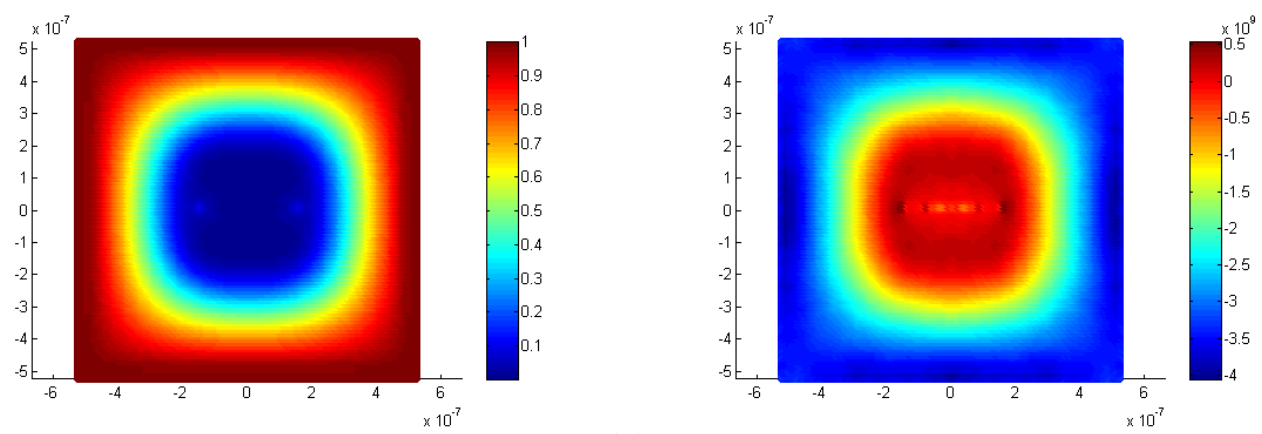

(c)

(d)

Figure 6. Results for an electrode plate with a single crack: (a) Initial damage (b) Damage after deformation (c) Lithium ion concentration (d) Hydrostatic stress

\subsection{Coupled analysis for multiple crack cases}

Due to unexpected factors such as manufacturing quality and damage occurance during transportation, electrodes may have multiple pre-existing cracks. Hence, single crack may not be sufficient for describing the condition of damage in a battery electrode. In these case studies, an electrode plate with multiple cracks with different orientations are investigated.

\subsubsection{Twin horizontal cracks}

A pair of cracks which are parallel to each other lie horizontally at the centre of a plate as shown in Fig. (7a). These cracks have the same length equivalent to $10 \%$ of the length of the specimen plate. Distance between these two cracks is $5 \%$ of the plate width. As lithiation progresses, the hydrostatic stress and lithium ion concentration increase at the crack tips. Once peridynamic bonds reach the critical stretch value, they will be broken and crack will propagate. As shown in Fig. (7b), upper crack propagates in upward direction and lower crack propagates in downward direction. Besides, according to Fig. (7d), high hydrostatic stress also exists for particles between these twin cracks, which means two cracks have a potential to merge into one large crack. 

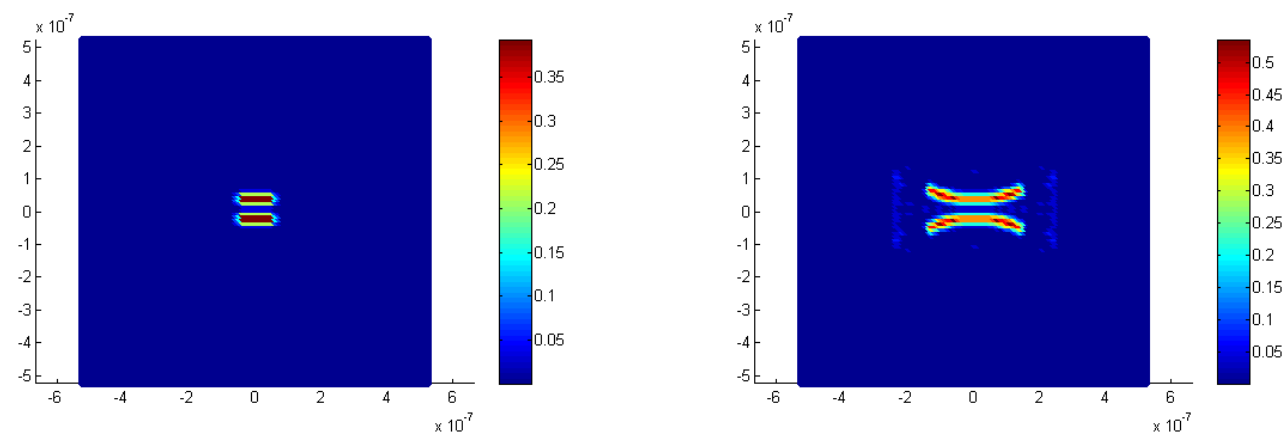

(a)

(b)
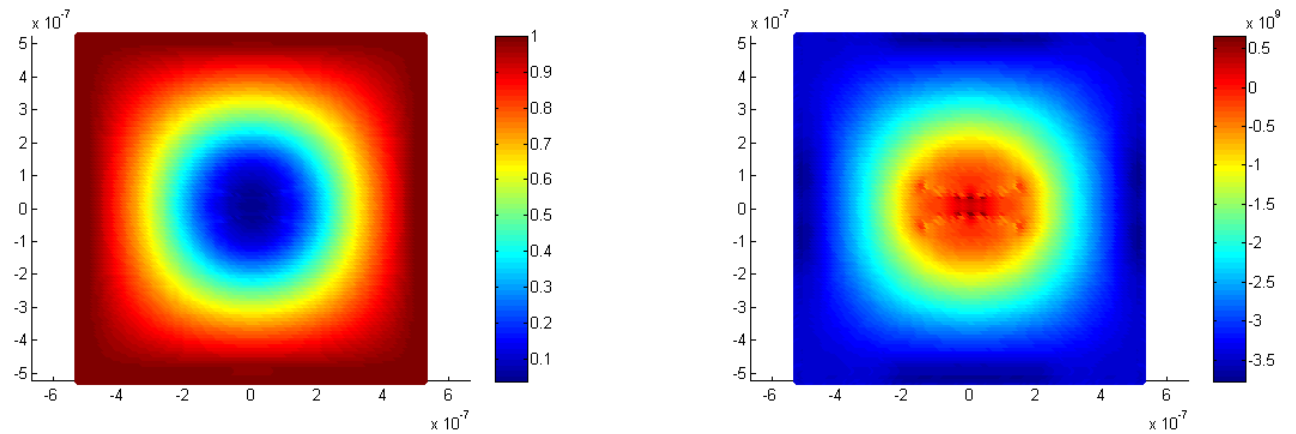

(c)

Figure 7. Results for an electrode plate with twin cracks: (a) Initial damage (b) Damage after deformation (c) Lithium ion concentration (d) Hydrostatic stress

\subsubsection{Perpendicular and oblique crack cases}

In the following case, two perpendicular cracks are located at the central region of specimen plate as shown in Fig. (8a). The length of the horizontal crack is $12 \%$ of the plate length and the size of the vertical crack is $10 \%$ of the plate length. The distance between the horizontal crack and upper tip of the vertical crack is $10 \%$ of the plate length. Once stretch of bonds reach critical value during lithiation process, the horizontal crack propagates upward first and then downward while the vertical crack simply propagates along the original crack path as shown in Fig. (8b). High hydrostatic stress lies at the crack tips and between the orthogonal cracks. These two cracks have a potential to merge into a large " $\mathrm{T}$ " shape crack. For more general situation, these two cracks can be arranged with an arbitrary angle as shown in Fig. (9a). Once these cracks merge with each other, the hydrostatic stress of the particles between cracks reduces as shown in Fig. (9d). 

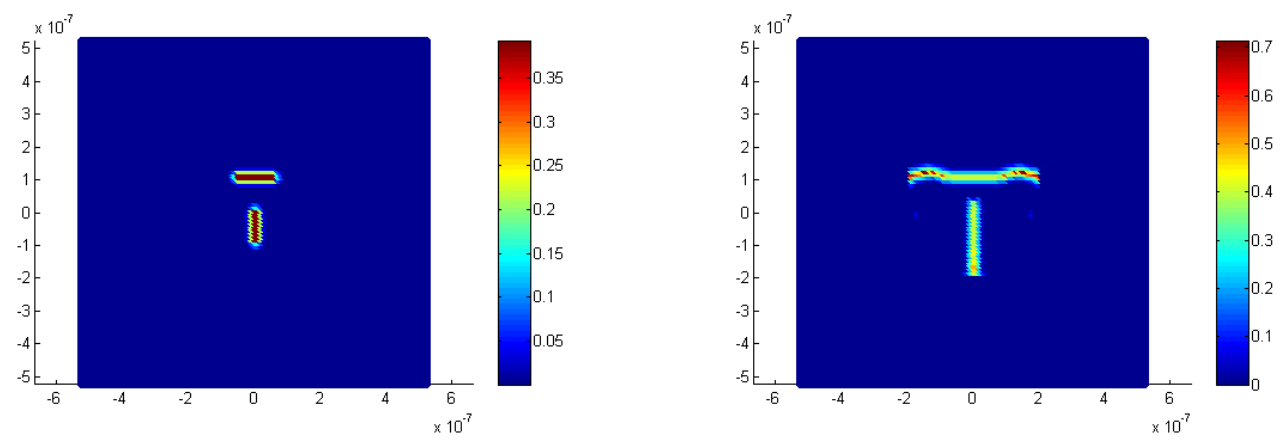

(a)

(b)
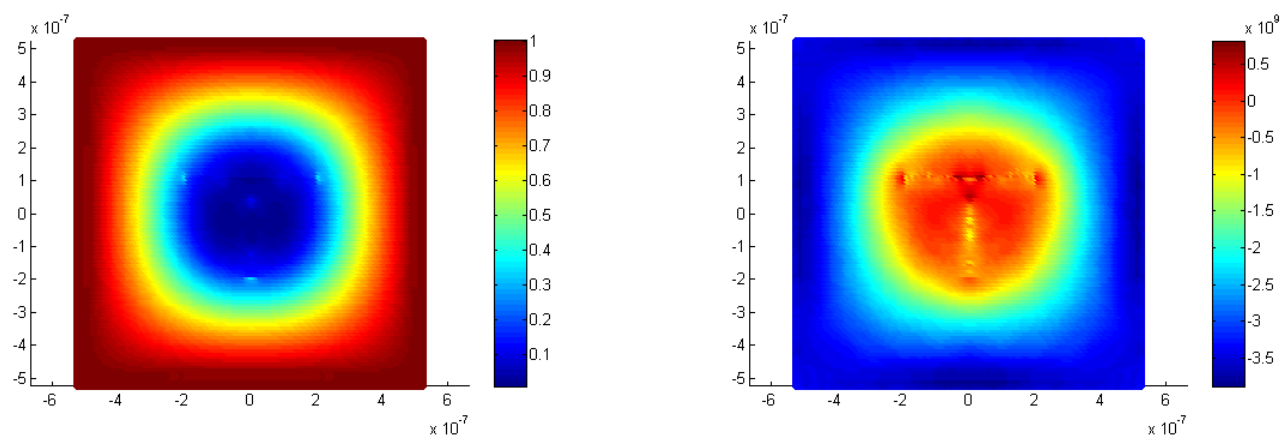

(c)

(d)

Figure 8. Results for an electrode plate with perpendicular cracks: (a) Initial damage (b) Damage after deformation (c) Lithium ion concentration (d) Hydrostatic stress

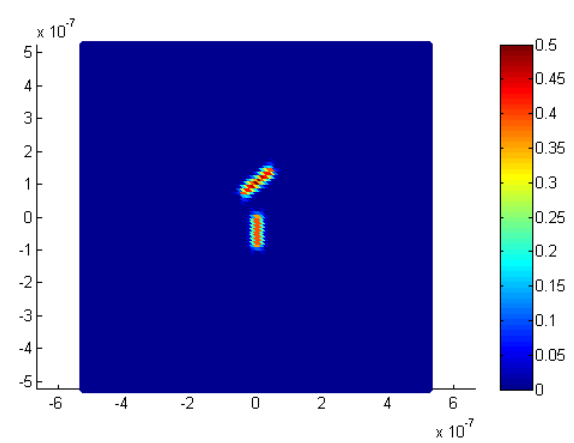

(a)
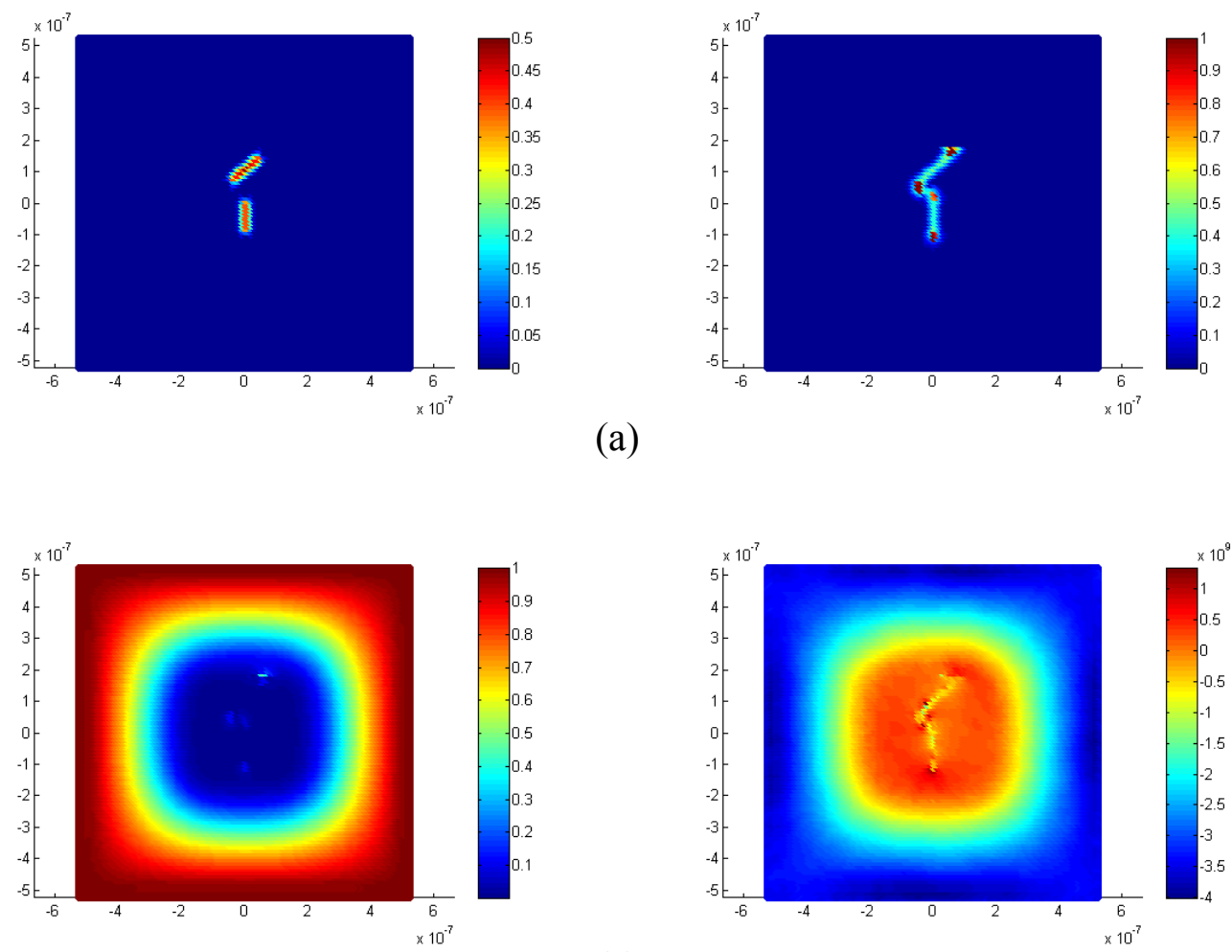

(b)

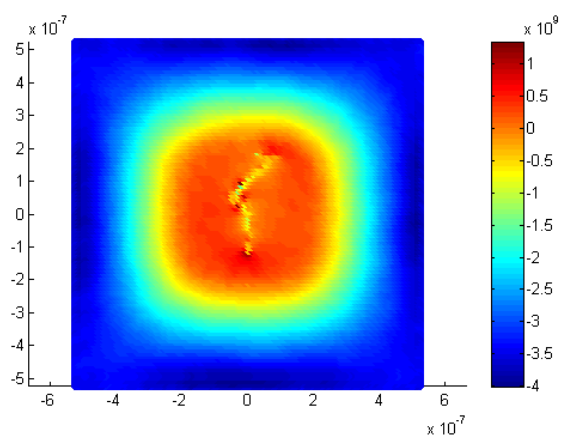

(c) (d) 
Figure 9. Results for an electrode plate with oblique cracks: (a) Initial damage (b) Damage after deformation (c) Lithium ion concentration (d) Hydrostatic stress

\subsubsection{Triple horizontal crack case}

Cracks in battery electrode may have arbitrary arrangements in terms of crack numbers and crack orientations. In this case, a triple crack damage situation is under investigation. Cracks with the same length equivalent to $10 \%$ of the plate length located at the central plate region are shown in Fig. (10a). Since cracks are located close to each other, particles between cracks have fewer interactions with neighbouring particles within their horizons. Therefore, the stiffnesses of these particles are relatively weaker. Hence, propagation of some cracks is led by neighbouring cracks. Central crack propagates towards neighbouring cracks from both crack tips and merges all three cracks into one crack as shown in Fig. (10b). Then, the propagation of outer crack tips continues horizontally along its initial crack path. High hydrostatic stresses exist at outer crack tips and the stresses at inner crack tips reduce since cracks already merge with each other as shown in Fig. (10d).
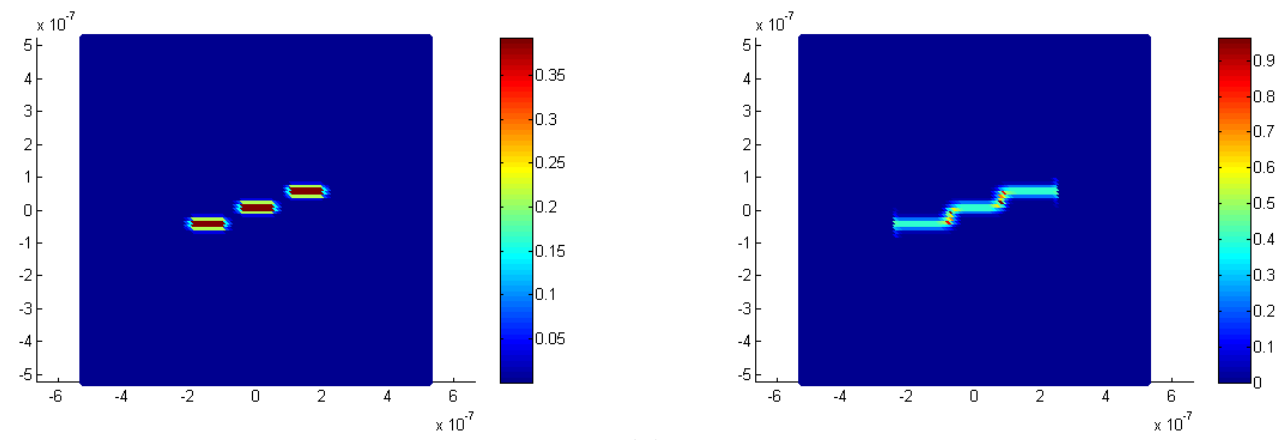

(a)
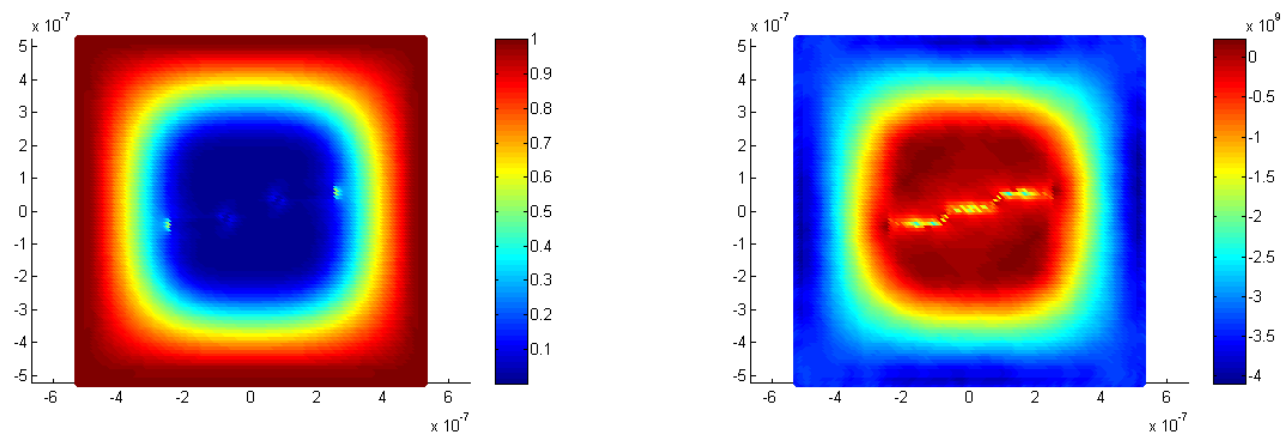

(c)

Figure 10. Results for an electrode plate with triple cracks: (a) Initial damage (b) Damage after deformation (c) Lithium ion concentration (d) Hydrostatic stress

\subsection{Coupled analysis for randomly oriented multiple crack case}

After certain cycling processes, lithium ion battery electrodes may accumulate large number of cracks. The size and orientation of these cracks depend on the structural design of the battery electrode plate [16]. In order to simulate the effect of damage on 
electrode plate after several cycling process, coupled field diffusion analysis for battery electrode with randomly oriented multiple crack cases are under investigation in this section. Two damage situations with six cracks and eleven cracks were chosen as shown in Fig. (11a) and Fig. (12a). All cracks have the same crack length equivalent to $10 \%$ of the specimen length and they are randomly oriented at the central plate region. As lithium-ions diffuse into crack tip regions, hydrostatic stress increases dramatically at the crack tips, especially at the outer crack tips. Meanwhile, some cracks start to propagate and merge with neighbouring cracks. Once small cracks merge into a large crack, hydrostatic stress at crack tip regions reduce which may be the reason that the remaining small cracks stop propagating. In other words, the newly formed large crack prevents small cracks to propagate. On the other hand, outer crack tips will not be affected by other cracks and propagate along their crack paths toward high concentration region.
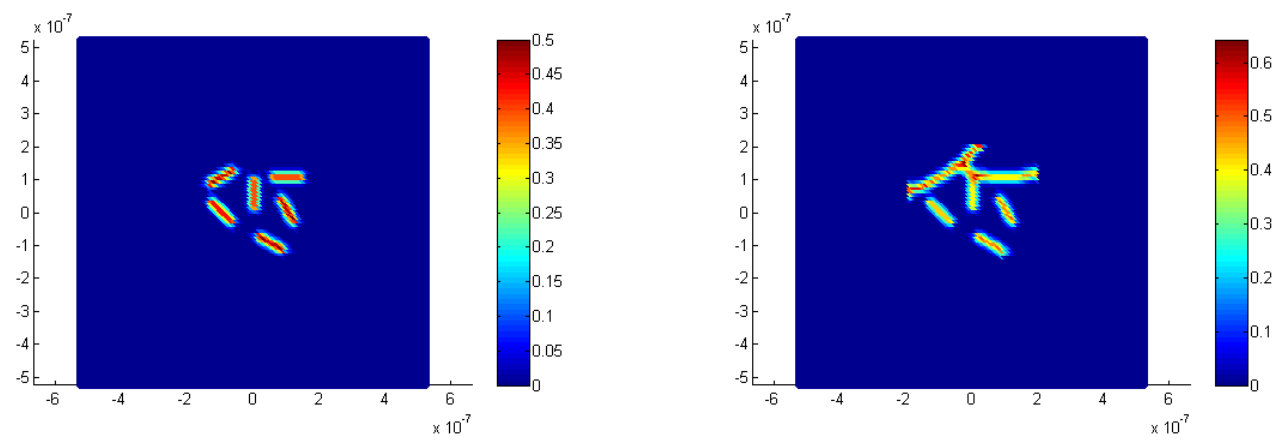

(a)

(b)
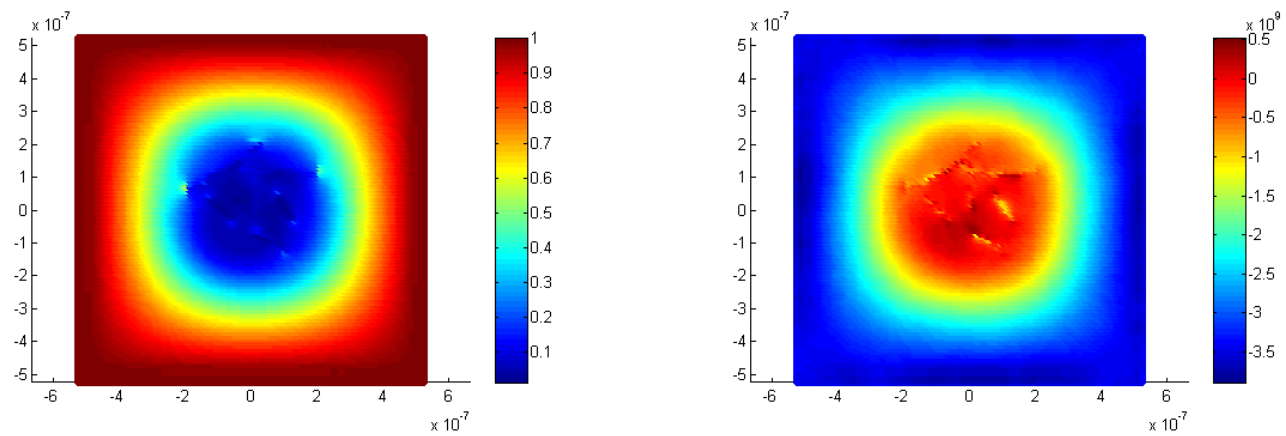

(c)

Figure 11. Results for an electrode plate with six randomly oriented cracks: (a) Initial damage (b) Damage after deformation (c) Lithium ion concentration (d) Hydrostatic stress 

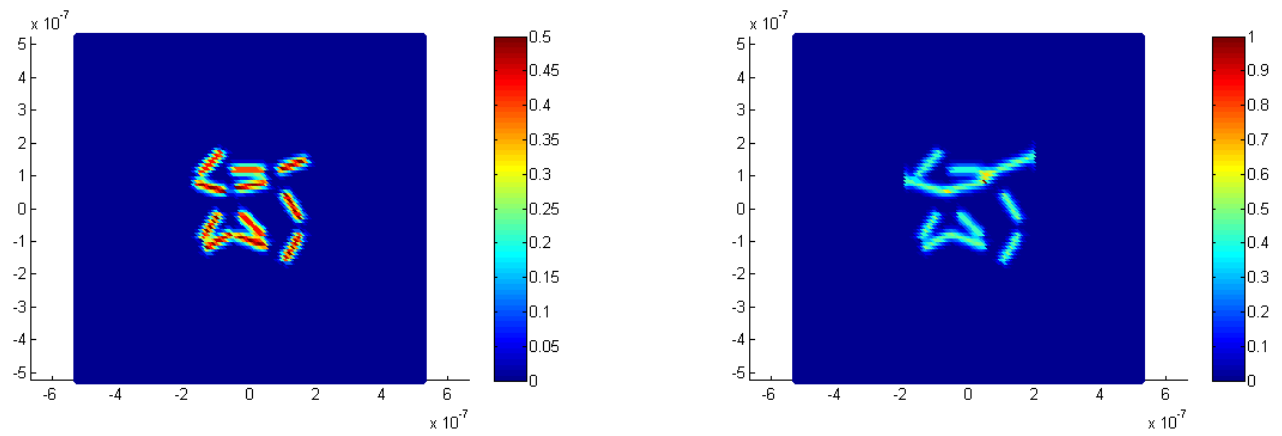

(a)

(b)
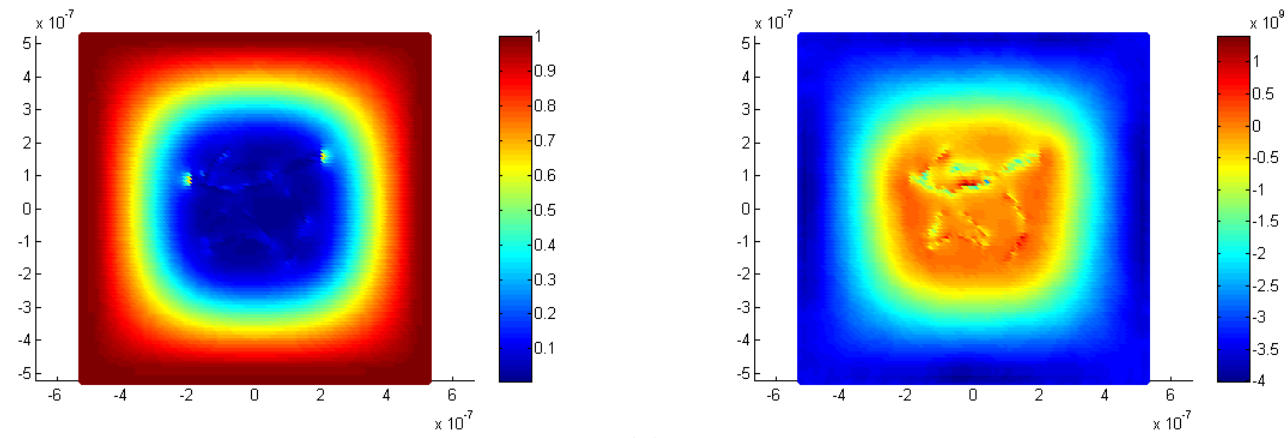

(c)

Figure 12. Result for an electrode plate with eleven randomly oriented cracks: (a) Initial damage (b) Damage after deformation (c) Lithium ion concentration (d) Hydrostatic stress

\section{Conclusion}

In this study, a new continuum mechanics formulation, peridynamics, in conjunction with the newly developed peridynamic differential operator approach is utilised to analyse the fracture and failure analysis of lithium-ion battery plates with pre-existing cracks. According to the numerical results, high hydrostatic stress at the crack tip is the main motivation of crack propagation and it is reflected on bond breakage in peridynamic theory. Besides, phase change from pure silicon and lithiated silicon during the lithiation process also affects crack propagation. As hydrostatic stress increases at the crack tip region, lithium-ion concentration increases as well and leads to higher concentration value at the crack tip regions. Although lithium-ion battery electrode may have various damage situations, cracks normally propagate through particles with weak stiffness and high hydrostatic stress. Hence, for multi-crack situations, some of the cracks merge into a larger crack first and then propagate from outer crack tips. Due to the crack merging process, high hydrostatic stress at some of the crack tips reduces and this may arrest remaining cracks.

As a summary, peridynamic theory can provide a good estimation of damage evolution in lithium ion battery plates. It is possible to obtain information about crack propagation without remeshing and using sophisticated damage criteria. By using 
peridynamics, we can have a better understanding on failure mechanisms in lithium ion batteries.

\section{References}

1. Daniel, C., 2008. Materials and processing for lithium-ion batteries. Jom, 60(9), pp.43-48.

2. Chan, C.K., Peng, H., Liu, G., McIlwrath, K., Zhang, X.F., Huggins, R.A. and Cui, Y., 2008. High-performance lithium battery anodes using silicon nanowires. Nature nanotechnology, 3(1), pp.31-35.

3. Maranchi, J.P., Hepp, A.F. and Kumta, P.N., 2003. High capacity, reversible silicon thin-film anodes for lithium-ion batteries. Electrochemical and solidstate letters, 6(9), pp.A198-A201.

4. Choi, J.W., Cui, Y. and Nix, W.D., 2011. Size-dependent fracture of Si nanowire battery anodes. Journal of the Mechanics and Physics of Solids, 59(9), pp.1717-1730.

5. Liu, P., Sridhar, N. and Zhang, Y.W., 2012. Lithiation-induced tensile stress and surface cracking in silicon thin film anode for rechargeable lithium battery. Journal of Applied Physics, 112(9), p.093507.

6. Stamps, M.A. and Huang, H.Y.S., 2012, November. Mixed modes fracture and fatigue evaluation for lithium-ion batteries. In ASME 2012 International Mechanical Engineering Congress and Exposition (pp. 97-103). American Society of Mechanical Engineers.

7. Grantab, R. and Shenoy, V.B., 2012. Pressure-gradient dependent diffusion and crack propagation in lithiated silicon nanowires. Journal of the Electrochemical Society, 159(5), pp.A584-A591.

8. Zuo, P. and Zhao, Y.P., 2015. A phase field model coupling lithium diffusion and stress evolution with crack propagation and application in lithium ion batteries. Physical Chemistry Chemical Physics, 17(1), pp.287-297.

9. Gao, Y.F. and Zhou, M., 2013. Coupled mechano-diffusional driving forces for fracture in electrode materials. Journal of Power Sources, 230, pp.176-193.

10. Madenci, E., Barut, A. and Futch, M., 2016. Peridynamic differential operator and its applications. Computer Methods in Applied Mechanics and Engineering, 304, pp.408-451.

11. Shenoy, V.B., Johari, P. and Qi, Y., 2010. Elastic softening of amorphous and crystalline $\mathrm{Li}-\mathrm{Si}$ phases with increasing Li concentration: a first-principles study. Journal of Power Sources, 195(19), pp.6825-6830.

12. Zhang, X., Lee, S.W., Lee, H.W., Cui, Y. and Linder, C., 2015. A reactioncontrolled diffusion model for the lithiation of silicon in lithium-ion batteries. Extreme Mechanics Letters, 4, pp.61-75. 
13. Silling, S.A. and Askari, E., 2005. A meshfree method based on the peridynamic model of solid mechanics. Computers \& structures, 83(17), pp.1526-1535.

14. Oterkus, S., Madenci, E. and Agwai, A., 2014. Fully coupled peridynamic thermomechanics. Journal of the Mechanics and Physics of Solids, 64, pp.1-23.

15. Malvern, L.E., 1969. Introduction to the Mechanics of a Continuous Medium (No. Monograph).

16. Li, J., Dozier, A.K., Li, Y., Yang, F. and Cheng, Y.T., 2011. Crack pattern formation in thin film lithium-ion battery electrodes. Journal of The Electrochemical Society, 158(6), pp.A689-A694.

17. Oterkus, S., Madenci, E., Oterkus, E., Hwang, Y., Bae, J. and Han, S., 2014, May. Hygro-thermo-mechanical analysis and failure prediction in electronic packages by using peridynamics. In Electronic Components and Technology Conference (ECTC), 2014 IEEE 64th (pp. 973-982). IEEE.

18. Zimmermann, M., 2005. A continuum theory with long-range forces for solids (Doctoral dissertation, Massachusetts Institute of Technology).

19. Silling, S.A., 2000. Reformulation of elasticity theory for discontinuities and long-range forces. Journal of the Mechanics and Physics of Solids, 48(1), pp.175-209.

20. Ren, H., Zhuang, X., Cai, Y. and Rabczuk, T., 2016. Dual-horizon peridynamics. International Journal for Numerical Methods in Engineering, 108(12), pp.1451-1476.

21. Dipasquale, D., Zaccariotto, M. and Galvanetto, U., 2014. Crack propagation with adaptive grid refinement in 2D peridynamics. International Journal of Fracture, 190(1-2), pp.1-22.

22. Diyaroglu, C., Oterkus, E., Madenci, E., Rabczuk, T. and Siddiq, A., 2016. Peridynamic modeling of composite laminates under explosive loading. Composite Structures, 144, pp.14-23.

23. Oterkus, E. and Madenci, E., 2012. Peridynamic theory for damage initiation and growth in composite laminate. In Key Engineering Materials (Vol. 488, pp. 355-358). Trans Tech Publications.

24. De Meo, D., Diyaroglu, C., Zhu, N., Oterkus, E. and Siddiq, M.A., 2016. Modelling of stress-corrosion cracking by using peridynamics. International journal of hydrogen energy, 41(15), pp.6593-6609.

25. Oterkus, S., 2015. Peridynamics for the solution of multiphysics problems. PhD Thesis, The University of Arizona. 
26. Madenci, E. and Oterkus, E., 2014. Peridynamic theory and its applications. New York: Springer.

27. Silling, S.A., Epton, M., Weckner, O., Xu, J. and Askari, E., 2007. Peridynamic states and constitutive modeling. Journal of Elasticity, 88(2), pp.151-184.

28. Fick, A., 1995. On liquid diffusion. Journal of Membrane Science, 100(1), pp.33-38.

29. Oterkus, S., Madenci, E. and Agwai, A., 2014. Peridynamic thermal diffusion. Journal of Computational Physics, 265, pp.71-96. 